\title{
Germanica
}

\section{L'idylle familiale ou la guerre des couples dans les récits de Birgit Vanderbeke}

Eine Familienidylle oder der Krieg zwischen den Geschlechtern in den Erzählungen von Birgit Vanderbeke

\section{Elfie Poulain}

\section{(2)enEdition}

\section{Journals}

Édition électronique

URL : http://journals.openedition.org/germanica/332

DOI : $10.4000 /$ germanica.332

ISSN : 2107-0784

\section{Éditeur}

Université de Lille

\section{Édition imprimée}

Date de publication : 1 décembre 2006

Pagination : 135-148

ISBN : 2-913857-18-3

ISSN : 0984-2632

Référence électronique

Elfie Poulain, «L'idylle familiale ou la guerre des couples dans les récits de Birgit Vanderbeke», Germanica [En ligne], 39 | 2006, mis en ligne le 19 février 2010, consulté le 06 octobre 2020. URL : http://journals.openedition.org/germanica/332 ; DOI : https://doi.org/10.4000/germanica.332

Ce document a été généré automatiquement le 6 octobre 2020.

(c) Tous droits réservés 


\title{
L'idylle familiale ou la guerre des couples dans les récits de Birgit Vanderbeke
}

\author{
Eine Familienidylle oder der Krieg zwischen den Geschlechtern in den \\ Erzählungen von Birgit Vanderbeke
}

Elfie Poulain

1 Birgit Vanderbeke est l'une des auteures à succès de la jeune génération d'écrivains de langue allemande. Le Dîner de moules ${ }^{1}$ lui a valu le prix Ingeborg Bachmann à Klagenfurt et lui a fait faire son entrée sur la scène littéraire. Depuis, elle a publié une dizaine d'ouvrages pour lesquels elle a également obtenu des prix littéraires². Née dans une famille de musiciens en 1956 dans le Brandebourg, elle s'enfuit à l'Ouest avec sa famille, juste avant la construction du Mur en 1961 et s'établit à Francfort-sur-le-Main. Elle y fait ses études de littérature et de droit, passe quelques mois à Berlin en 1992 avant de quitter l'Allemagne en 1993 pour le Sud de la France où elle vit depuis de sa propre plume. Dès son premier livre, elle trouve son thème de prédilection :

"Je raconte ", dit-elle en 1998, « le désarroi entre les hommes parce que pour moi, c'est le thème le plus fascinant au monde. Les gens, dès qu'ils sont les uns avec les autres, sont souvent aussi les uns contre les autres, et cela les mène à des situations lamentables $»^{3}$.

2 Sans être autobiographiques, ses récits s'inspirent de la réalité vécue et reflètent, sur le mode imaginaire, une réalité à laquelle ils sont dialectiquement articulés. Ils reprennent les thèmes de ses modèles littéraires : Uwe Johnson, Max Frisch et Ingeborg Bachmann, mais Birgit Vanderbeke les traite sur un ton différent, un ton léger et enjoué, qui lui permet de mettre en évidence les mentalités qui génèrent ces conflits. Avec humour et ironie, elle décrit le quotidien familial comme le lieu par excellence où s'expriment les rapports conflictuels et où se pense la hiérarchie des sexes afin de faire réfléchir sur l'éternelle et double image de l'amour et de la guerre entre hommes et femmes. 
3 Les trois récits choisis se lisent comme une chronique de l'idylle familiale, ou de ce qui en reste, à travers deux générations. Le Dîner de moules met en scène la figure du père dans son rôle de despote familial, dans Guerre froide $e^{4}$, c'est la mère qui impose un régime non moins tyrannique à sa petite famille, et dans Alberta reçoit un amant ${ }^{5}$, elle peint une jeune femme en quête d'autonomie, qui se présente comme la digne héritière de la petite fille ou de l'adolescente rebelle des récits précédents. Ces récits nous livrent ce que promet leur titre tout en basculant dans son contraire : le Dîner de moules n'a pas lieu, les temps paisibles (titre allemand) marque un temps de guerre, et Alberta reçoit bien son ancien amant, mais c'est pour l'éconduire. Ils constituent une mise en question ironique des rôles traditionnels de la femme et de l'homme dans la société occidentale.

\section{Le Dîner de moules ou la gloire déchue du patriarcat}

La narratrice du récit, une jeune fille d'environ 18 ans, son jeune frère et leur mère attendent vers 6 heures du soir le père qui doit revenir d'un voyage d'affaires. Pour fêter sa promotion, la dernière sur le chemin de son succès professionnel, ils lui ont préparé les moules dont il raffole. Or, contre toute habitude, il n'arrive pas à l'heure prévue. Aussi, la famille se met-elle à parler de sa vie, et surtout de lui, du grand absent qui occupe alors l'avant-scène de cette narration. Le craquement des moules qui s'ouvrent dans la bassine choque et dégoûte la fille. Il accompagne comme image métaphorique les révélations choquantes du drame intérieur de leur famille, celui de la domination et de l'oppression intolérable du père dont chacun d'eux avait souffert en silence.

5 Pour ce père, cadre supérieur d'une grande entreprise, la profession et la famille sont une priorité absolue. Il se voit comme un homme intelligent et rationnel qui trouve la logique abstraite très belle. Et il l'applique pour construire ce qu'il appelle « une vraie famille» :

tout le monde devait se transformer quand mon père rentrait à la maison, pour que nous fassions tous une vraie famille, comme disait mon père, parce qu'il n'avait pas eu de famille, mais qu'il s'était fabriqué une idée très précise de ce qu'était une vraie famille, et il pouvait devenir ultrasusceptible quand on ne s'y conformait pas ${ }^{6}$ (DM 26-7).

6 La mère s'y conformait et mettait toujours pour l'accueillir, au grand dam de sa fille, « son visage des soirées en famille », qui relayait « son visage d'école » qu'elle prenait le matin pour enseigner dans son lycée, et celle " de femme au foyer », ce visage crevé et épuisé que les enfants lui connaissaient quand elle s'esquintait pour la famille. La logique du père s'étendait à tous les aspects de leur vie, à leurs goûts et à leurs pensées. Contrairement aux autres, il détestait les disciplines littéraires et artistiques comme quelque chose d'inutile qui ne met aucun moteur en marche. Seule la musique faisait exception. Dans une vraie famille, disait-il, les enfants doivent apprendre à jouer un instrument. Il fallait s'exercer une heure par jour, pas plus, et surtout pas quand il rentrait fatigué de son travail. La mère, qui aurait bien voulu devenir musicienne, n'avait pas le droit de jouer un instrument car le père détestait son côté sentimental. C'est pourquoi, après leur fuite, elle garda son violon caché dans l'armoire froide de la chambre à coucher jusqu'au jour où elle le retrouva cassé. Très rarement, quand elle était triste, dit la fille, elle jouait du piano et chantait des lieder de Schubert, tout Le 
Voyage d'hiver aller et retour, en pleurant copieusement (cf. DM 35). Pour le père, la musique était réservée à l'abonnement aux concerts et au dimanche matin, où il écoutait le Chœur des esclaves en compagnie de ses enfants, condamnés à rester au salon. La mère qui n'aimait pas écouter cette calamité acoustique, ce banal schroum schroum des contrebasses, s'enfermait dans la cuisine pour préparer le rôti. Elle n'en ressortait que quand Verdi avait quitté le séjour pour aérer la pièce afin d'expulser les restes du Trouvère (cf. DM 68). Elle craignait qu'avec la promotion du père, il n'y eût plus d'abonnement aux concerts car, une fois devenu cadre dirigeant, le père ne serait plus tenu de se faire voir à ces concerts par tous les cadres supérieurs.

7 Bien que condition nécessaire, la musique de Verdi n'était toutefois pas suffisante pour que le dimanche soit réussi ${ }^{7}$. Il fallait encore que le rôti soit bon et qu'il n'y ait pas de bouchons quand ils partaient prendre l'air ensemble. Car dans une vraie famille, il fallait tout faire ensemble, regarder le soir la télé ensemble, passer obligatoirement les vacances ensemble et obligatoirement, au sud, au bord de la mer, là où il $\mathrm{y}$ avait beaucoup de soleil. La mère et les enfants qui étaient très sensibles au soleil devaient profiter le plus possible de ce soleil pour lequel ils avaient payé si cher. Aussi, pour calmer leurs brûlures, le père leur mettait-il du jus de citron, et la fille de dire qu'ils n'avaient jamais su ce qui était pire, les coups de soleil avec ou sans jus de citron (cf. DM 76). Côté argent, la logique du père était également d'un genre particulier. D'origine extrêmement modeste, ce qui n'était pas le cas de la mère, il avait horreur de l'odeur des pauvres, exigeait tous les jours une nappe blanche, propre et repassée, donnait des pourboires gigantesques et portait des costumes sur mesure. La mère, bien qu'elle eût son salaire de professeur, se voyait contrainte d'acheter ses robes dans des magasins à fins de série. Autant dire que le père n'aimait pas se montrer avec elle en public. C'est que le père avait une logique abstraite alors que la mère avait seulement un sens pratique, concret, explique la fille.

8 La logique du père s'appliquait surtout à l'usage du langage, dont il faisait son instrument de domination. On l'écoutait parler, lui seul. Il racontait des histoires de bureau à la mère tandis que la mère ne racontait jamais au père des histoires d'école parce que le bureau était important et valait mieux que l'école (cf. DM 74, M 59). Si le père écoutait les autres, c'était pour mettre de l'ordre dans sa famille. Il avait institué un système de surveillance réciproque et les faisait venir, le soir, un à un dans le salon pour se faire donner la version des faits pendant que les autres attendaient dehors :

À la fin, il tirait des conclusions logiques, puis fixait et exécutait les punitions, et effectivement, on avait tous assez peur, car les punitions étaient fixées en vertu de

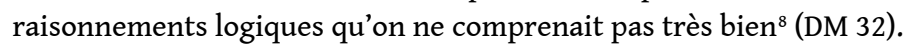

Somme toute, ils ne disaient plus ce qu'ils pensaient, ils ne disaient que ce que le père voulait entendre. La mère elle-même n'osait rien dire car, dit la fille, elle avait un respect immense pour le père parce qu'il était biologiste et que cela valait mieux que d'être un bel esprit.

9 Le tour de force de la fille rebelle consista à amener le frère et la mère, le soir de ce Dîner, à se livrer à des confidences et à faire exploser de la sorte le système familial des dénonciations, qui n'était pas sans rappeler le système politique de la surveillance du citoyen par le citoyen, de l'ancienne RDA. La narration est d'autant plus convaincante que la fille $y$ imite avec une exactitude glaciale le ton et le style du père. L'humour qui en résulte fait tout le plaisir de ce texte et génère la distance critique nécessaire pour démasquer la tyrannie du père. Avec une logique tout aussi implacable que celle du père, la fille juxtapose les paroles et les actions de ce dernier pour faire voir son jeu 
d'être et de paraître. La reconnaissance commune de son ambivalence explique la décision tacite de la famille de ne plus se soumettre à sa loi et de ne pas décrocher le téléphone lorsqu'il sonne, à 10 heures du soir.

La soirée racontée n'est ni plus, ni moins qu'une guerre de libération, à huis-clos. L'attitude de chacun à l'égard de la musique est particulièrement révélatrice de l'attitude qu'ils ont à l'égard de la famille. Si le chœur des prisonniers fait le délice du père et que la mère adore l'harmonie classique, la fille, elle, affiche un goût tout différent :

je trouvais l'harmonie classique franchement suspecte avec ses dominantes et ses sous-dominantes, je soupçonnais cette harmonie d'être un simple fourre-tout, je disais à ma mère, les pauvres voix, on les fourre brutalement dans cette harmonie ${ }^{9}$ (DM 120).

Comment ne pas voir dans cette remarque une métaphore de la hiérarchie familiale, d'autant plus que la fille dit préférer la musique dodécaphonique dans laquelle, comme nous le savons, la hiérarchie des notes est abolie? Conformément à l'image traditionnelle des rôles, le père y dicte sa loi comme Dieu avait dicté sa loi à la nature, pour se soumettre femme et enfants comme Adam avait été appelé à se soumettre la nature ${ }^{10}$. Et la mère, bien qu'elle exerce le métier d'enseignante qui lui permet de remplir ses engagements à l'égard de la famille au même titre que le père, continue à vivre entièrement dans une dynamique d'adaptation et de soumission. Comme femme elle est rejetée dans une position d'infériorité systématique face au mari qui se présente comme être de raison et qui tire sa force de la faiblesse de son épouse, complice, malgré elle, de son bourreau. À la fin, la mère avoue son admiration pour Médée, qui tua ses enfants pour se venger de son mari. Elle avait donc nourri une opposition dans son for intérieur, mais l'intériorisation du rôle traditionnel de la femme l'a forcée à la refouler. La mutation des rôles ne s'amorce qu'avec la fille : elle aura le courage d'opposer son jugement personnel à ces lois patriarcales.

\section{Guerre froide ou la lutte amère du matriarcat}

11 Ce récit nous offre une perspective inter-allemande ${ }^{11}$. Il se situe chronologiquement à une date antérieure, dans les années 60 . Cette fois, c'est la mère qui tyrannise son monde avec autant de raffinement que ne le faisait le père dans le récit précédent. Les trois enfants y doivent encore une obéissance inconditionnelle aux parents. Après avoir fui l'ancienne RDA, cette famille s'est installée à l'Ouest. Bien qu'appartenant au passé, la guerre continue à y opposer les parents comme la Guerre froide opposait alors l'Est et l'Ouest. La narratrice, une fillette de 6 ans environ, raconte la peur qui en émane pour les enfants en rapportant, avec toute la naïveté de son âge, les formules toutes faites du père et de la mère. Elle confronte ainsi le monde des enfants au monde énigmatique des adultes. Ce procédé confère au récit une double structure et une ironie hilarante. Elle écrit :

Ce qui plaisait à mon père, à l'Est, c'est qu'ils avaient essayé le socialisme, et ce qui ne lui plaisait pas, c'est qu'ils n'avaient pas réussi à le faire marcher parce qu'ils étaient tous idiots. Cela, nous le savions. Ce qui lui plaisait à l'Ouest, nous l'ignorions. Ce qui plaisait à ma mère à l'Ouest, c'est que, au lieu de sa mère, c'était elle maintenant qui pouvait faire disparaitre les clefs. C'est en tout cas ce que disait le père ${ }^{12}$ (GF 7). 
Cette mère au foyer qui gère et contrôle absolument tout est entièrement préoccupée par les affaires de la famille comme l'étaient traditionnellement les femmes de notre société jusqu'au milieu du $\mathrm{xx}^{\mathrm{e}}$ siècle. C'est elle qui décide de faire disparaitre les clés des toilettes et de couper le chauffage en avançant de bonnes raisons : il fallait vérifier la digestion des enfants, la chaleur pourrait favoriser la présence des microbes, etc. Le père, lui, ne se souciait pas de ces détails, mais s'indignait du chauffage coupé car, disait-il, ce n'était par pour geler comme à l'Est qu'ils étaient partis à l'Ouest. Quant aux filles, pour échapper au froid, elles avaient un jour commencé à utiliser les toilettes de l'école, qui étaient chauffées. La narratrice écrit :

Je crois que la guerre qui régnait dans l'appartement était liée à cette histoire de clefs, parce que le père a fini lui aussi par utiliser des toilettes et une salle de bains ailleurs, et, lorsqu'il a commencé cela, la mère a dit en pleurant, je ne vivrai pas vieille, c'est sûr, je vais mourir jeune à tous les coups, et dès lors, elle a eu Wasa et moi de son côté, bien que sur le fond, nous fussions plutôt du côté de notre père ${ }^{13}$ (GF 9).

Quand le père revient tard dans la nuit ou encore le lendemain, fraîchement baigné et parfumé, elle entend la mère pleurer. Une de ces nuits, la mère avait tiré les enfants hors du lit, les avait habillés à la hâte en mettant leurs habits à l'envers, et avait pris la voiture avec eux. Arrivée sur un pont, elle leur avait déclaré qu'ils allaient maintenant sauter dans le Rhin, mais la narratrice, prise de panique, s'était jetée sur le volant, l'avait tourné dans une autre direction et les avait ainsi sauvés de la catastrophe.

Le voyage jusqu'au Rhin et la plainte qu'elle allait mourir jeune font vivre les enfants sous la pression permanente de la peur de sa mort. Aussi essayent-ils de lui causer le moins de soucis possible. Mais, dit la narratrice, ils avaient beau prendre toutes les précautions du monde, dès qu'un souci était éliminé, il s'en présentait aussitôt un autre à l'horizon. Le père, lui aussi, avait fait des efforts après le voyage jusqu'au Rhin et sortait parfois avec la mère. Mais comme il aimait les soirées copieusement arrosées et qu'il tenait malgré tout à conduire la voiture, ces sorties s'achevaient par des disputes. Les enfants, restés seuls à la maison, redoutaient le pire et se mettaient à penser au voyage jusqu'au Rhin.

14 Aussi, les petites guerres ne s'arrêtaient-elles pas et les enfants se mirent à réfléchir à l'éventualité d'un divorce et aux conséquences qu'il pouvait avoir pour eux :

le père, avec ses secrets, ne risquait que sa femme, alors que nous, nous risquions notre mère, et il nous semblait clair qu'une mère vaut plus pour ses enfants qu'une femme pour son mari, qui peut toujours s'en trouver une autre. Les enfants ne peuvent pas se trouver une nouvelle mère, une fois que la première est dans la tombe, et les belles-mères des enfants ne valent pas mieux que celles des maris, nous savions ce qu'il fallait penser des premières par les enfants des Osterloh et des secondes par le père ${ }^{14}$ (GF 63).

Comme le père avait commencé à acheter des disques américains, de la musique nègre, disait leur mère, elles pensaient qu'il leur mijotait une grosse maman nègre. L'avantage d'une telle deuxième mère, imaginaient-elles, pouvait être un barbecue le dimanche comme le faisaient les mamans américaines de l'autre côté du terrain vague. La constellation inverse, un beau-père amerloque, leur semblait peu probable car déjà un beau-père de l'Ouest tout ce qu'il y a de plus normal était exclu depuis que leur mère leur avait dit à quelle sorte de femmes elle appartenait (cf. GF 136-7). Aussi le divorce leur semblait-il augmenter le risque de voir leur mère "mourir jeune " : pouvaient-ils compter jusqu'à 30 ans, ou à 40 , ce qui leur laissait encore un peu de temps? Les 
parents pouvaient-ils les couper en deux comme on l'avait fait pour les deux Lottchen ${ }^{15}$ ? Un soir, en pleine époque américaine (cf. GF 91), la mère partie à une réunion de parents d'élèves, le père les amène voir un des meilleurs films hollywoodiens ${ }^{16}$ auquel les enfants ne comprirent que ce qui touchait à leur drame familial :

La seule chose que nous étions sûres d'avoir vue et comprise, c'était l'histoire de la femme d'origine et de la femme de rechange, et il était clair que le père voulait être comme l'homme dans le film [.... ${ }^{17}$ (GF 93).

Cet épisode, l'un des plus touchants, illustre tout l'art de Birgit Vanderbeke de raconter des histoires à double fond pour faire voir les scènes de guerre du film et celles que vivent ces enfants à la douce lumière de l'ironie.

Guerre froide s'achèvera avec une nouvelle réconciliation et une nouvelle guerre autour de la maison à construire. Aussi fictionnel que soit ce récit, il éclaire à la lumière crue de la vérité l'un des problèmes sociaux de notre monde contemporain : la menace de la dissolution des mariages. Dans ce récit, le code culturel agit encore comme un frein pour éviter les ruptures définitives, mais il ne saurait préserver des traumatismes qu'engendrent de tels conflits pour les enfants et les adultes. Les deux parents manifestent la mentalité de l'époque. Le père, bienveillant à l'égard des siens, se donne des libertés dont jouissaient traditionnellement les hommes, et la mère, inquiète à l'extrême, s'avère incapable de voir qu'elle provoque elle-même les infidélités de son époux. Sans position sociale hors de la maison, elle se replie sur elle-même et intériorise le conflit vécu en développant une attitude masochiste. Comme l'écrit Freud :

Le refoulement de l'agressivité de la femme lui est constitutionnellement prescrit et socialement imposé, il favorise le développement des impulsions masochistes [...] Le masochisme est donc, comme on dit, typiquement féminin ${ }^{18}$.

Pour compenser, elle développe une fixation du souci pour l'autre, pour les enfants, qui lui permet de refouler le potentiel d'agressivité qu'elle développe à l'égard de son mari. Mais en même temps, son amour féminin exclusif dégénère en amour-combat et la pousse vers une auto-destruction qui fait souffrir ses enfants.

\section{Alberta reçoit un amant ou le désir d'autonomie}

Les deux récits analysés démasquent la détermination fondamentale des êtres humains à vivre en fonction des valeurs de force ou de faiblesse, à devenir bourreaux ou victimes, et à rester tributaires d'une image socioculturelle réglant le cliché masculin et féminin. Ce $3^{\mathrm{e}}$ récit, qui eut un grand succès, montre une évolution des mentalités et des rapports entre hommes et femmes. Les conditions sociales et historiques ayant changé avec les générations, on s'aperçoit que les lois sur l'égalité, valides uniquement en droit, sont devenues réalité ou presque dans le vécu quotidien d'Alberta. Son comportement tranche nettement avec celui de la génération de sa mère. Chez elle, il n'est plus question d'autodépréciation, ni de la soumission qui a historiquement affecté les femmes ${ }^{19}$. Alberta est une femme émancipée, autonome dans ses pensées, qui se fait reconnaître dans sa dimension personnelle. Mais son histoire montre que l'homme et la femme continuent à ne pouvoir se passer l'un de l'autre, que la recherche du bonheur et de l'amour reste inchangée et que la guerre des couples n'en est pas pour autant terminée. La narratrice constate :

Il est étonnant, au fond, ai-je pensé, que les humains soient si mal équipés pour affronter une chose aussi sérieuse, susceptible, qui plus est, d'arriver à tout le 
monde ou presque à un moment donné, et qui arrive même plus d'une fois à certains. [...] En réalité, personne ne comprend rien à l'amour, même si tout le monde prétend le contraire et peut débiter au moins trois ou quatre théories à ce sujet. Mais, dès que ça devient sérieux, on s'aperçoit tout de suite que les théories ne tiennent pas debout parce que justement votre cas personnel n'y figure pas, on n'y trouve jamais que des modèles simples, et les cas personnels ne sont pas simples mais uniques et compliqués [...] Il devrait y avoir des cours d'autodéfense pour vous prémunir contre cette chose unique, ai-je pensé20 (AA 91-92).

Le récit est structuré en 3 chapitres : le $1^{\text {er }}$ chapitre relate le malentendu sur l'amour entre Alberta et Nadan, le $2^{\mathrm{e}}$ parle des relations compliquées entre la narratrice et son mari Jean-Philippe, et au $3^{\mathrm{e}}$ chapitre, le lecteur découvre qu'Alberta est l'alter ego de la narratrice elle-même. Le récit déborde de comique et pourtant, il y va des choses des plus sérieuses, de la vie et de l'amour, et de la question inquiétante de savoir pourquoi les choses vont toujours mal. L'image sur la couverture de l'édition allemande, une femme qui se retourne, illustre le regard rétrospectif de la narratrice qui commence son récit quelques jours avant l'Ascension avec Alberta et Nadan qui veulent fuguer, quitter tout pour vivre ailleurs leur grand amour devant lequel ils ont déjà capitulé en vain plusieurs fois. Leur première rencontre eut lieu alors qu'Alberta avait 15 ans et que Nadan s'apprêtait à passer son permis de conduire. Ce premier soir au clair de lune, Alberta attendit en vain son premier baiser. Depuis, chacun d'eux a continué sa route jusqu'au jour où, durant leurs études, leurs chemins se croisent à nouveau et qu'ils font le projet d'un avenir commun, pensant découvrir une fois de plus qu'ils s'aiment depuis toujours et jusqu'à leur dernier jour (cf. AA 29). L'idée de leur fugue tient à ce que la maison que Nadan avait fait construire pour eux les mettait très mal à l'aise. Or, aussitôt partis, leurs divergences se manifestent et ils s'irritent l'un l'autre. Aussi, après une première nuit dans un hôtel tout proche de l'autoroute, reconnaissent-ils la faillite de leur amour éternel et la narratrice constate ce qu'elle a toujours su, au fond: que l'amour dans la tête est beaucoup plus simple que l'amour dans la vie (cf. AA 46). Ils se séparent à nouveau, et Nadan partira avec une bourse post-doctorale en Arizona tandis qu'Alberta sera lectrice à Lyon.

20 C'est ainsi que s'achève le premier volet de l'histoire à laquelle succède celle de la narratrice, mariée à Jean-Philippe depuis 8 ans, mère d'une petite fille et traductrice de profession. Malgré l'infidélité de son mari qu'elle feint d'ignorer, elle continue à écrire son récit sur Alberta et Nadan, comme Jean-Philippe le lui avait suggéré. Mais un jour, un coup de téléphone ébranle son équilibre précaire : c'est Nadan qui s'annonce, 20 ans après leur première rencontre, pour dire qu'il souhaite la revoir. Émue comme au premier jour, elle s'apprête à le recevoir tout en ruminant leur passé, et notamment une chose qu'elle aurait préféré oublier, le jour où, après son avortement, elle avait appelé Nadan à l'aide et que celui-ci lui avait lancé un « Non » glacial. Elle se dit :

mais maintenant c'était là et cela faisait mal et me rappelait que les guerres entre hommes et femmes sont bien des vraies guerres aussi. Quand j'étais jeune, je ne croyais pas que c'étaient de vraies guerres où l'un peut conduire l'autre à sa perte, j'étais étonnée en apprenant dans les livres les étranges blessures que l'amour peut vous infliger, j'ignorais tout des mutilations et des morts que les hommes et les femmes peuvent se faire subir mutuellement ${ }^{21}$ (AA 105-6).

Cependant, elle l'accueille dans un esprit serein, l'écoute parler de ses enfants et de sa femme enceinte, sans pouvoir s'empêcher de penser que 20 ans auparavant, ils avaient été assis à table, exactement comme maintenant, pour s'imaginer leur avenir commun. 
Mais lorsqu'il cherche à placer sa main sur la sienne, elle se lève brusquement pour le congédier.

21 À comparer les couples des trois récits, force est de constater des ressemblances et des dissemblances dans le comportement des deux générations. Nadan reste tout proche du cliché masculin traditionnel. C'est un esprit rationnel, volontaire, qui vise avant tout son succès professionnel et qui dit : «Je ne fais que ce que je veux vouloir» (AA 80) ${ }^{22}$. En Arizona déjà, après sa séparation d'Alberta, il s'était adonné corps et âme à son travail même si, occasionnellement, il se surprenait à regarder des boucles d'oreilles dans une vitrine. Son retour en Europe n'avait pas été motivé par Alberta, mais par l'effondrement de sa thèse qui avait fait sa renommée dans le milieu scientifique là-bas (cf. AA 64, A 62). Quant à sa vie privée, il la planifie aussi rationnellement que sa profession. La maison, par exemple, qu'il avait fait construire pour Alberta sans jamais lui demander ses vœux n'était qu'une objectivation pure et simple de ses désirs égocentriques de femme et de famille. Comme Alberta, qui n'était plus la douce Dulcinée d'autrefois, ne s'y était pas conformée, il avait épousé une femme qui correspondait à son image de femme, une femme instrument qui lui garantissait la reproduction de soi et lui conférait une solidité sociale. À Alberta, il dira : Il était tout simplement temps de fonder une famille (cf. AA 114, A 113). Toutefois, le désir d'amour ne l'avait pas quitté pour autant. Dans sa tête, il visait à concilier l'amour conjugal, dissocié du désir, et l'amour passion qu'il venait chercher auprès d'une maîtresse, d'Alberta en l'occurrence. Il tentait ainsi de vivre la vie double dont, tout jeune, il avait parlé à Alberta (cf. AA76).

Son comportement ne se distingue guère de l'image masculine héritée du passé. Celle d'Alberta, par contre, est nettement plus nuancée car elle assume son rôle de femme et de mère sans se vouloir assujettie à un homme. Elle est le sujet de ses propres actes et décide elle-même du sens qu'elle veut donner à sa vie. Jeune, elle a mené une vie indépendante à Lyon et elle a assumé seule la décision de l'avortement. Cet acte, devenu légal depuis la loi Veil en 1974, représente un grand tournant dans l'histoire des hommes et des femmes. À l'époque des mères, il était difficile de vivre avec un homme sans être mariée, et encore moins d'avoir un enfant naturel. Qu'on se rappelle la mère dans Le Dîner de moules : elle avait dû se marier parce qu'elle était enceinte. À cette époque encore, la femme était complètement dépendante de sa nature, et la nature était ce que voulait le mari. L'homme était le maitre de la procréation, la pierre de voûte de l'autorité familiale. Il n'en est plus ainsi pour Alberta qui se fait reconnaître comme femme-sujet sans passer par la médiation de l'homme et par la mise au monde des enfants. Sa décision d'avoir un enfant ou d'adopter une attitude conciliante à l'égard de son mari n'est pas dictée par une contrainte matérielle ou sociale, c'est un acte de jugement personnel et libre.

Mais nonobstant cette autonomie manifeste, on reconnaît en elle des paramètres qui ont toujours été attachés à la femme. Elle reste fidèle à la vocation maternelle et éduque et soigne la petite Cécile toute seule alors que le père prend ses plaisirs à Lyon. Et quand elle vit sa désillusion avec Nadan, elle manifeste une grande sensibilité émotive. Elle dit :

chez les femmes, le chagrin d'amour touche tout de suite la personne entière et la paralyse (AA 66) [...] un homme et une femme, dès qu'ils font quelque chose ensemble, fût-ce la chose la plus anodine de la terre, entendent et vivent des réalités absolument différentes et n'arrivent pas à se mettre d'accord, après coup, sur ce qu'ils ont vu, entendu et vécu' ${ }^{23}$ (AA 95). 
Aussi, même si elle vit sur le mode de l'être de raison, comme les hommes, elle reste toujours un être de sentiment, à savoir une femme.

Bilan :L'image des relations familiales tracée dans les récits de Birgit Vanderbeke fait état d'une profonde mutation dans la conception des rôles sociaux. Si le père dans Le Dîner de moules apparaît encore comme un Tartuffe de nos jours qui détruit ce qu'il veut créer, à savoir une vraie famille, Alberta, elle, sonne le glas d'une vie familiale construite sur le modèle patriarcal où la femme est considérée comme la moitié soumise et imparfaite de l'homme, et les enfants comme de simples objets à domestiquer. La vie familiale n'y est plus scindée par une hiérarchie d'essence où la femme se définit comme inférieure par nature, mais elle montre deux êtres humains dont le rapport se fonde sur la réciprocité et le respect mutuel, autrement dit sur l'idée de l'égalité. Cette idée qui nous vient des Lumières a provoqué de longs débats au cours des siècles derniers avant de devenir de nos jours une réalité ${ }^{24}$. Les écrits littéraires de Birgit Vanderbeke se lisent comme une réflexion sociologique sur le chemin parcouru par notre société en vue de trouver une vraie culture de partage, mais ils montrent aussi que la construction de la vie à deux est devenue de nos jours des plus complexes dans sa réalisation. G. Fraisse cite Sénancour qui

affirme que si les filles font les mêmes choses que les garçons, si les femmes ont les mêmes droits que les hommes, si l'identité entre les hommes et les femmes supplante leur différence, l'amour est détruit au profit de l'amitié, ce qui est terrible.

Et elle lui oppose Stendhal qui dit :

on n'empêchera jamais le rossignol de chanter au printemps ${ }^{25}$.

Les récits de Birgit Vanderbeke étayent ce constat et ils nous montrent que la recherche de l'âme sœur continue à exister et que l'amour a encore de beaux jours devant lui.

\section{NOTES}

1. Birgit Vanderbeke, Das Muschelessen, Francfort sur-le-Main : Fischer Tb, 14 éd. 2004 ( $1^{\text {re }}$ éd. Berlin : Rotbuch Verlag, 1990), tr. fr. par Claire de Oliveira Le Dîner de moules, éd. Stock, 1995. L'ouvrage sera cité sous le sigle $\mathrm{M}$ et DM.

2. Le prix Kranichstein en 1997, le prix de Soluthurn en 1999 et le Prix Hans Fallada en 2002.

3. Cité par Richard Wagner, in Ich hatte ein bißchen Kraft drüber. Zum Werk von Birgit Vanderbeke, Francfort sur-le-Main : Fischer Tb, 2001, p. 9: «Ich erzähle von der Verwirrung zwischen den Menschen, weil sie für mich das Allerspannendste auf der Welt ist. Daß die Menschen, wenn sie miteinander sind, oft gegeneinander sind und daß dies dann zu jämmerlichen Situationen führt ».

4. Birgit Vanderbeke, Friedliche Zeiten, Francfort sur-le-Main : Fischer TB, $3^{\text {e }}$ éd. 2001 ( $1^{\text {re }}$ éd. Berlin: Rotbuch Verlag, 1996), tr. fr. Guerre froide, tr. par Anne Weber, Paris: Stock, 1997. L'ouvrage sera cité sous le sigle FZ et GF. 
5. Birgit Vanderbeke, Alberta empfängt einen Liebhaber, Francfort sur-le-Main : Fischer TB, $6^{\mathrm{e}}$ éd. 2003 ( $1^{\text {re }}$ éd. Berlin : Alexander Fest Verlag, 1997), tr. fr. Alberta reçoit un amant, tr. par Anne Weber, Paris : Stock, 1997. L'ouvrage sera cité sous le sigle A et AA.

6. M 20 : « es mussten sich ja alle umstellen, wenn mein Vater nach Hause kam, damit das Ganze eine richtige Familie war, wie mein Vater das nannte, weil er keine Familie gehabt hat, dafür hat er die genauesten Vorstellungen davon entwickelt, was eine richtige Familie ist, und er hat ausgesprochen empfindlich werden können, wenn man dagegen verstieß ».

7. La logique du père est basée sur les concepts des rationalistes qui distinguent les raisons nécessaires et les raisons suffisantes. Cf. Leibniz, Théodicée et Kant, Critique de la raison pure.

8. M 25 : «Am Schluß hat er logische Schlüsse gezogen und danach die Strafen festgesetzt und ausgeführt, und in Wirklichkeit haben wir alle ziemliche Angst gehabt, weil die Strafen nach logischen Schlussfolgerungen festgesetzt worden sind, die keiner so richtig begreifen hat können ».

9. M 96 : « überhaupt habe ich gesagt, mir ist die klassische Harmonie mitsamt ihren Dominanten und Subdominanten äußerst suspekt, ich habe den Verdacht gehabt, dass alles in diese Harmonie nur hineingequetscht würde ».

10. Cf. La Genèse 2, 22, cf. Epître aux Romains 7, 2.

11. Cf. Raimund Kagerer, "Geschichte einer Entwurzelung ", in Ich hatte ein bißchen Kraft drüber, op. cit., p. $254:$ : mit einem Wort, ein deutsch-deutsches Kabinettstückchen ».

12. FZ, 5 : « Mein Vater mochte am Osten, daß sie es mit dem Sozialismus versucht hatten, und er mochte am Osten nicht, daß sie den Sozialismus nicht zum Laufen gebracht hatten, weil sie alle Idioten waren. Soviel wussten wir. Was er am Westen mochte, wussten wir nicht ; Meine Mutter mochte am Westen, daß jetzt sie die Schlüssel verschwinden lassen konnte und nicht mehr ihre Mutter. Das jedenfalls sagte der Vater ».

13. FZ 6: «Ich glaube, der ganze Krieg in dieser Wohnung hing mit der Schlüsselgeschichte zusammen, weil schließlich der Vater auch anfing, woanders das Klo und das Badezimmer zu benutzen, und als er damit anfing, sagte die Mutter weinend : Ich werde bestimmt mal nicht alt, ich sterbe bestimmt mal jung, und von dem Moment an hatte sie Wasa und mich auf ihrer Seite, obwohl wir in der Sache selbst eher auf der Seite unseres Vaters waren [...] ».

14. FZ 55 : "weil der Vater mit seinen Geheimnissen bloß seine Frau aufs Spiel setzte, wir hingegen unsere Mutter, und uns war klar, daß eine Mutter für ihre Kinder mehr wert ist als eine Frau für ihren Mann, der immerhin eine neue finden kann. Kinder können keine neue Mutter finden, wenn die erste einmal im Grab ist, und von Stiefmüttern war ungefähr so viel zu halten wie von Schwiegermüttern, das eine wußten wir von den Osterloh-Kindern und das andere vom Vater $[. .]$.$» .$

15. Il s'agit du livre d'Erich Kästner, Das doppelte Lottchen, 1949 (Deux pour une).

16. Cf. GF 99, FZ 89 : « einer der besten Hollywoodschinken ».

17. FZ 83 : «Das einzige, was wir sicher gesehen und verstanden hatten, war die Sache mit der Erst- und der Zweitfrau, und es war klar, daß unser Vater so wie der Mann im Film sein wollte $[\ldots] »$.

18. Cf. Sigmund Freud, «Die Weiblichkeit », in Gesammelte Werke 1, Francfort sur-le-Main : Fischer Tb, 1982, p. 547.

19. Pour Rousseau, «L femme et l'homme sont fait l'un pour l'autre [...]. Ainsi toute l'éducation des femmes doit être relative aux hommes. Leur plaire, leur être utiles, se faire aimer et honorer d'eux. » In Émile ou l'éducation, Paris, 1964, p. 455.

20. À 89-90 : «Es ist im Grunde ganz erstaunlich, dachte ich, wie schlecht die Menschen gar öfter. [...] Mit der Liebe kennt sich in Wirklichkeit keiner aus, obwohl es jeder behauptet und mindestens drei oder vier Theorien dazu hersagen kann. Aber wenn es ernst wird, merkt man sofort, daß die Theorien nichts taugen, weil ausgerechnet der eigene Fall nicht darin vorkommt, 
sondern immer nur schlichte Modelle, und die eigenen Fälle sind nicht schlicht, sondern einmalig und kompliziert [...] Es müßte, dachte ich, Selbstverteidigungskurse dagegen geben ». 21. À 104 : «aber nun war es da und tat weh und erinnerte mich daran, daß Kriege zwischen Männern und Frauen schließlich auch richtige Kriege sind. Als ich jung war, glaubte ich nicht, daß es richtige Kriege sind, in denen einer den anderen ins Verderben schicken kann, ich staunte, wenn ich in Büchern von den seltsamen Versehrungen las, die man durch Liebe davontragen kann, ich wußte nichts von den Verstümmelungen und Toden, die Männer und Frauen einander antun können [...]». Cette réflexion rappelle la problématique du cycle Todesarten de I. Bachmann que Birgit Vanderbeke aborde ici tout autrement.

22. À 79 : «Ich tue nur, was ich auch wollen will».

23. À 65 : «bei Frauen ist immer gleich der ganze Mensch von Liebeskummer betroffen und lahmgelegt ». À 93 : "weil ein Mann und eine Frau, sobald sie auch nur irgend etwas, und sei es das Belangloseste auf der Welt, zusammen machen, absolut etwas Verschiedenes sehen und hören und erleben und sich später nie mehr darauf einigen können, was sie gesehen und gehört und erlebt haben [...]».

24. Cf. Geneviève Fraisse, "La différence des sexes, une différence historique ", in La Controverse des sexes, Paris : PUF, 2001, p. 13-38.

25. Cf. in G. Fraisse, op. cit., p. 50.

\section{RÉSUMÉS}

Les récits de Birgit Vanderbeke, déjà étudiés dans les écoles allemandes, présentent les caractéristiques de la nouvelle génération d'écrivains allemands, tant pour l'humour décapant et stimulant qui tranche avec l'écriture de la génération précédente que pour le regard neuf qu'elle porte sur la société de notre temps. Notre analyse abordera trois de ses récits pour dégager l'un de ses thèmes majeurs : la relation conflictuelle entre les êtres et la question de la hiérarchie des sexes. Elle tentera de montrer la mutation profonde que l'auteur discerne dans l'image des rôles de l'homme et de la femme qui mène notre société du système patriarcal traditionnel à une culture de partage basée sur les concepts de l'autonomie et de l'égalité.

Birgit Vanderbekes Erzählungen, die bereits Schullektüre geworden sind, weisen die typischen Kennzeichen der neueren deutschsprachigen Literatur auf, den zugleich scharfen und stimulierenden Humor, der im Gegensatz zur vorangehenden Schriftstellergeneration steht, und den Blick der jungen Generation auf die Probleme unserer gegenwärtigen Gesellschaft. Unsere Analyse von drei Erzählungen der Autorin befaßt sich mit einem ihrer Hauptthemen, den konfliktreichen zwischenmenschlichen Beziehungen und der Frage nach der Hierarchie der Geschlechter. Sie soll den tiefgreifenden Wandel aufzeigen, den die Autorin hinsichtlich des sozialen Rollenbildes von Mann und Frau wahrnimmt und der unsere traditionell patriarchale Gesellschaft zu einem auf Autonomie und Gleichheit beruhenden gesellschaftlichen System führt. 
INDEX

Mots-clés : couple

oeuvrecitee Das Muschelessen, Le dîner de moules, Friedliche Zeiten, Guerre froide, Alberta empfängt einen Liebhaber

\section{AUTEURS}

\section{ELFIE POULAIN}

Université Charles-de-Gaulle - Lille 3 